\title{
A Practical Synchronization Approach for Memristive Hyperchaotic System
}

\author{
Meng Hui*, Yincheng Zhang, Ning Han and Ni Luo \\ School of Electronics and Control, Chang'an University, Middle Section of 2nd Ring Road, Xi'an, 710064, China \\ ${ }^{*}$ Corresponding author
}

\begin{abstract}
Chaotic synchronization has great potential applications in engineering, physics and mathematics. Memristive hyperchaotic system synchronization is a hot spot for synchronization study. A practical synchronization approach is used in this paper. Compared with previous synchronization methods, this approach does not need the error of driven system and response system to be zero when the time goes to infinity. Detailed mathematical proof of this synchronization method is given to verify the electiveness. Finaly, a memristive hyperchaotic system is used to test the synchronization approach and the simulation results indicate that this method is elective.
\end{abstract}

Keywords—synchronization; memristive; chaotic systems

\section{INTRODUCTION}

A hyperchaotic attractor is typically defined as chaotic behavior with at least two positive Lyapunov exponents. Combined with one null exponent along the flow and one negative exponent to ensure the boundness of the solution. The first hyperchaotic attractor was proposed by Rossler [1] in 1979, then hyperchaotic systems have became one of the research hotspots due to its potential applications in many fields of interest. The minimal dimension for a continuous hyperchaotic system is four and it presents a higher level of complexity. Hyperchaotic system could be used in diverse applications such as secure communications [2,3], neural networks [4], nonvolatile random access memory(NRAM) [5], or laser design [6].

Chua introduced the missing fourth circuit element, in 1971 [7]. However, until 2008 Hewlett-Packard Labs realized a solid-state thin film two terminal memristor. Till now, the study of memristive system is hot, apply memristive system to realize hyperchaotic system is a very new research topic. Zhou proposed a memristive hyperchaotic system by replacing the resistor in modified Lv multiwing system [8]. Then, Ma proposed a memristive hyperchaotic system by adding a common smooth ux-controlled memristor and a cross product item into a 3D pseudo four-wing chaotic system [9], and the system can only exhibit four-wing attractor, and needs an extra cross-product item.

Synchronization is ubiquitous in Nature. The pioneer work of chaos synchronization was first reported by Pecora and Carroll [10] in 1990. Since then, synchronization of chaotic systems have been widely studied. Many methods have been designed to synchronize chaotic systems, such as active control [11], adaptive control [12] and so on. However, all aforementioned works amid of chaotic synchronization based on Lyapunov theory and set the error of driven system and response system to be zero when the time goes to infinity. In fact, in real physical systems or experimental situations noise or disturbance may exist, thus, it is very difficult to control the error to be zero for actual system. This paper used a new synchronization approach for hyperchaotic systems synchrionzation. This method doesn't need strictly limit the synchronization error to be zero.

\section{MAIN RESULTS}

The driven system is set as below:

$$
x(t)=C x(t)+f(x(t))
$$

where $x(t)=\left(x_{1}(t), x_{2}(t), \cdots, x_{m}(t)\right)^{T} \in R^{m} \times 1$ is the state vectors. $C \in R^{m} \times m$ is a constant matrix, $f(x(t))$ is nonlinear function.

$$
y(t)=C y(t)+f(y(t))+\mathrm{U}(\mathrm{x}(\mathrm{t}), \mathrm{y}(\mathrm{t}))
$$

where $y(t)=\left(y_{1}(t), y_{2}(t), \cdots, y_{m}(t)\right)^{T} \in R^{m} \times 1$ is the state vectors, $U(x(t), y(t))$ is feedback controllers which will be designed later.

The error system between the drive system (1) and the response system (2) is defined as

$$
\mathrm{e}(t)=y(t)-\mathrm{x}(\mathrm{t})
$$

The driven system and response system is said to be synchronization if

$$
\lim _{t \rightarrow \infty}\|e(t)\|=\|y(t)-x(t)\|=0
$$

$\|\cdot\|$ denotes matrix norm. The equation (4) is the criteria often used in previous synchronization research work. However, this criteria is very difficult for practical application. In order to improve this situation, a new better synchronization approach is proposed. 
The driven system (1) and response system (2) is said to be $\delta$-synchronization if

$$
\lim _{t \rightarrow \infty}\|e(t)\|=\|y(t)-x(t)\| \leq \delta
$$

for an arbitrarily small value $\delta>0$.

Many previous synchronization studies researched the integral or fractional chaotic systems. These chaotic systems could be represented by polynomials. The nonlinear parts (denoted by $f(x(t))$ ) of polynomials could be rewritten as

$$
f(y(t))-f(x(t))=L(x(t)) e(t)+N(x(t), e(t))
$$

where $L(x(t)) e(t)$ and $N(x(t), e(t))$ represent the linear and nonlinear parts with respect to synchronization error $e(t)$. $L(x(t)) \in R^{m \times m}$ and $N(x(t), e(t)) \in R^{m \times 1}$ are real matrices. Now, let's introduce the projective $\delta$-synchronization criteria.

Theorem 1. Set the feedback controllers $U(x(t), y(t))$ in response system (2) be $U(x(t), y(t))=[D-L(x(t))] e(t)$. Assume the two conditions below hold, the driven system (1) and response system (1) is said to be $\delta$-synchronization.

(i) The initial value $\left.N(x(t), e(t))\right|_{e(t)=0}=0$, and $\lim _{e(t) \rightarrow 0} \frac{\|N(x(t), e(t))\|}{\|e(t)\|}=0$ for any $x(t)$.

(ii) $\frac{\pi}{2}<|\arg (C+D)| \leq \pi,(i=1,2, \cdots, m)$ and $\sigma=\|C+D\|>1$.

where $D \in R^{m \times m}$ is a constant matrix to be designed, $\frac{\pi}{2}<|\arg (C+D)| \leq \pi,(i=1,2, \cdots, m)$ is the eigenvalues of matrix $C+D$ and $\|C+D\|$ is the $l_{2}-$ norm of matrix $C+D$.

Proof Using the equation (6) and the designed controller $U(x(t), y(t))=[D-L(x(t))] e(t)$ the error of driven system (1) and response system (2) could be rewritten as

$$
e(t)=(C+D) e(t)+N(x(t), e(t))
$$

Based on the condition (i), we can get there exist a constant $K$ and $\eta>0$ so

$$
\|N(x(t), e(t))\|<\frac{\|e(t)\|}{K}
$$

for $\|e(t)\|<\eta$ and $t \geq 0$.

Considered the solution $e(t)$ in equation (7) for $\left\|\left.e(t)\right|_{t=0}\right\|<\eta_{0}\left(0<\eta_{0}<\eta\right)$, where $\eta_{0}$ is an arbitrarily small. Now, let's discuss the solution of $\delta$-synchronization error system. For the sake of simplicity, let matrix $M=C+D$ in the next parts of paper.Fistly, taking Laplace transform of equation (7), we can get

$$
s L[e(t)]-e(0)=M L[x(t)]+L[N(x(t), e(t))]
$$

where $e(0)$ is the initial condition. So

$$
L[e(t)]=\frac{s-M}{e}(t)+\frac{L[N(x(t), e(t))]}{s-M}
$$

Taken the inverse Laplace transform for (7) yield to

$$
e(t)=E_{1,1}(M t) e(0)+\int_{0}^{t} E_{1,1}(M(t-\tau)) N(x(\tau), e(\tau)) d \tau
$$

$\varepsilon_{0}\left(0<\varepsilon_{0}<\varepsilon\right)$ is a arbitrarily small constant, and consider the solution $e(t)$ for which $\|e(0)\|<\varepsilon_{0}$. Based on Generalized Gronwall inequality, equation (11) could be rewritten as

$$
\begin{aligned}
\|e(t)\| \leq & K \varepsilon_{0}[1+t\|M\|]^{-1} \\
& +\int_{0}^{t}[1+(t-\tau)\|M\|]^{-1}\|e(\tau)\| d \tau
\end{aligned}
$$

Considered the Lemma 2 the (|ref\{normet\}) could be written as

$$
\begin{aligned}
\|e(t)\| \leq & K \varepsilon_{0}[1+t\|M\|]^{-1}+\int_{0}^{t} \frac{K \varepsilon_{0}[1+\tau\|M\|]^{-1}}{[1+(t-\tau)\|M\|]^{1-(\|M\|)^{-1}}} d \tau \\
= & K \varepsilon_{0}\left\{[1+t\|M\|]^{-1}+\int_{0}^{t / 2} \frac{[1+\tau\|M\|]^{-1}}{[1+(t-\tau)\|M\|]^{1-(\|M\|)^{-1}}} d \tau\right. \\
& \left.+\int_{t / 2}^{t} \frac{[1+\tau\|M\|]^{-1}}{[1+(t-\tau)\|M\|]^{-\left(-(M \|)^{-1}\right.}} d \tau\right\}
\end{aligned}
$$

Since $t-\tau \geq \tau$ for $\tau \in[0, t / 2], t-\tau \leq \tau$ for $\tau \in[t / 2, t]$. Due to $\pi / 2<\left|\arg \lambda_{i}(M)\right| \leq \pi(i=1,2, \cdots n)$, and $\|M\|>1$, we could get,

$$
\begin{aligned}
& \|e(t)\| \leq K \varepsilon_{0}\left\{[1+t\|M\|]^{-1}+\int_{0}^{t / 2} \frac{[1+\tau\|M\|]^{-1}}{[1+\tau\|M\|]^{1-\|M\|^{-1}}} d \tau+\int_{t / 2}^{t} \frac{[1+(t-\tau)\|M\|]^{-1}}{[1+(t-\tau)\|M\|]^{1-\|M\|)^{-1}}} d \tau\right\} \\
& \leq K \varepsilon_{0}\left\{[1+t\|M\|]^{-1}+2 \int_{0}^{t / 2} \frac{[1+\tau\|M\|]^{-1}}{[1+\tau\|M\|]^{1-\|M\| \|^{-1}}} d \tau\right\} \\
& <K \varepsilon_{0}\left\{t\|M\|^{-1}+\frac{2\|M\|^{\left[[|M|)^{-1}-1\right]}}{1-\|M\|}\left(\frac{t}{2}\right)^{(1-\| M \mid)|| M \|}-\frac{2\|M\|^{\left.[|\| M|)^{-1}-1\right]}}{1-\|M\|}\right\}
\end{aligned}
$$

Consider $\|M\|>1$, when time $t \rightarrow+\infty$, 


$$
\begin{gathered}
\lim _{t \rightarrow+\infty}\|e(t)\|=\lim _{t \rightarrow+\infty} K \varepsilon_{0}\left\{t\|M\|^{-1}+\frac{2\|M\|^{\left[(\|M\|)^{-1}-1\right]}}{1-\|M\|}\left(\frac{t}{2}\right)^{(1-\|M\|) /\|M\|}\right. \\
\left.+\frac{2\|M\|^{\left[(\|M\|)^{-1}-1\right]}}{\|M\|-1}\right\} \\
=2 K \varepsilon_{0} \frac{\|M\|^{\left[\left(\|M\|^{-1}-1\right]\right.}}{\|M\|-1}<2 K \varepsilon_{0} \frac{1}{\|M\|-1}
\end{gathered}
$$

Set $\delta=2 K \varepsilon_{0} \frac{1}{\|M\|-1}$, the equation(15) becomes

$$
\lim _{t \rightarrow+\infty}\|e(t)\| \leq \delta
$$

Since $\varepsilon_{0}$ is an arbitrarily small, the constant $\delta$ be arbitrarily small. The driven system (1) and response system (2) have achieved $\delta$-synchronization base on the result of equation (16). This completes the proof.

\section{NUMERICAL RESULTS}

In this section, a memristive hyperchaotic systems is used to show the effectiveness of the approach.

Actually, different mathematical models of memristors have been proposed in $[13,14]$. In this paper, we used the memristor model developed in [15], which is described by a smooth quadratic nonlinear function as follows

$$
q(\varphi)=-a \varphi+0.5 b \varphi|\varphi|
$$

where $a$ and $b$ are both positive parameters and the memductance function is given by

$$
W(\varphi)=\frac{d q(\varphi)}{d \varphi}=-a+b|\varphi|
$$

where $a=1.5, b=1$.

Using this memristor, a hyperchaotic memristive system is constructed as

$$
\left(\begin{array}{l}
\dot{x}_{1}(t) \\
\dot{x}_{2}(t) \\
\dot{x}_{3}(t) \\
\dot{x}_{4}(t)
\end{array}\right)=\left(\begin{array}{cccc}
-4.8 & 7 & 0 & 0 \\
1 & -1 & 1 & 0 \\
0 & -10 & -0.11 & 0 \\
1 & 0 & 0 & 0
\end{array}\right)\left(\begin{array}{c}
x_{1}(t) \\
x_{2}(t) \\
x_{3}(t) \\
x_{4}(t)
\end{array}\right)+\left(\begin{array}{c}
x_{1}(t)\left|x_{4}(t)\right| \\
0 \\
0 \\
0
\end{array}\right)
$$

where $x_{4}$ denotes the $\varphi$ in memristor.The attractor of hyperchaotic memristive system is shown in Fig.1.

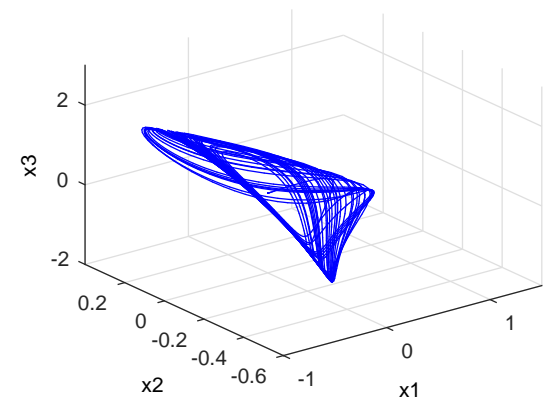

FIGURE I. THE ATTRACTOR OF HYPERCHAOTIC MEMRISTIVE SYSTEM

Let the system (19) be the driven system, also, the response system is given as

$$
\left(\begin{array}{l}
\dot{y}_{1}(t) \\
\dot{y}_{2}(t) \\
\dot{y}_{3}(t) \\
\dot{y}_{4}(t)
\end{array}\right)=\left(\begin{array}{cccc}
-4.8 & 7 & 0 & 0 \\
1 & -1 & 1 & 0 \\
0 & -10 & -0.11 & 0 \\
1 & 0 & 0 & 0
\end{array}\right)\left(\begin{array}{l}
y_{1}(t) \\
y_{2}(t) \\
y_{3}(t) \\
y_{4}(t)
\end{array}\right)+\left(\begin{array}{c}
y_{1}(t)\left|y_{4}(t)\right| \\
0 \\
0 \\
0
\end{array}\right)+U(x(t), y(t))
$$

It's obviously that the matrix

$$
C=\left(\begin{array}{cccc}
-4.8 & 7 & 0 & 0 \\
1 & -1 & 1 & 0 \\
0 & -10 & -0.11 & 0 \\
1 & 0 & 0 & 0
\end{array}\right)
$$

Based on the discussion in Part II, we can obtain

$$
f(y(t))-f(x(t))=\left(\begin{array}{cccc}
x_{4}(t) & 0 & 0 & x_{1}(t) \\
0 & 0 & 0 & 0 \\
0 & 0 & 0 & 0 \\
0 & 0 & 0 & 0
\end{array}\right) e(t)+\left(\begin{array}{c}
e_{1}(t) e_{4}(t) \\
0 \\
0 \\
0
\end{array}\right)
$$

Hence,

$$
L(x(t))=\left(\begin{array}{cccc}
x_{4}(t) & 0 & 0 & x_{1}(t) \\
0 & 0 & 0 & 0 \\
0 & 0 & 0 & 0 \\
0 & 0 & 0 & 0
\end{array}\right)
$$

and 


$$
N(x(t), e(t))=\left(\begin{array}{c}
e_{1}(t) e_{4}(t) \\
0 \\
0 \\
0
\end{array}\right)
$$

It can be easily get

$$
\lim _{e(t) \rightarrow 0}\|N(x(t), e(t))\|=0
$$

And

$$
\begin{aligned}
\lim _{e(t) \rightarrow 0} \frac{\|N(x(t), e(t))\|}{\|e(t)\|} & =\lim _{e(t) \rightarrow 0} \frac{\sqrt{\left(e_{1}(t) e_{4}(t)\right)^{2}}}{\sqrt{e_{1}^{2}(t)+e_{2}^{2}(t)+e_{3}^{2}(t)+e_{4}^{2}(t)}} \\
& =\lim _{e(t) \rightarrow 0} \frac{\sqrt{\left(e_{4}(t)\right)^{2}}}{\sqrt{1+\left(\frac{e_{2}(t)}{e_{1}(t)}\right)^{2}+\left(\frac{e_{3}(t)}{e_{1}(t)}\right)^{2}+\left(\frac{\left.e_{4}(t)\right)^{2}}{e_{1}(t)}\right.}} \\
& \leq \lim _{e(t) \rightarrow 0} \sqrt{\left(e_{4}(t)\right)^{2}}=0
\end{aligned}
$$

So, $\lim _{e(t) \rightarrow 0} \frac{\|N(x(t), e(t))\|}{\|e(t)\|}=0$. The condition(i) of Theorem is satisfied. Then, choose suitable matrix $D$ to satisfy condition (ii), the synchronization could be realized.

Let matrix

$$
D=\left(\begin{array}{cccc}
4.7 & -5 & 0 & 0 \\
-3 & 0.9 & -1 & 0 \\
0 & 10 & 0 & 0 \\
-1 & 0 & 0 & -2
\end{array}\right), C+D=\left(\begin{array}{cccc}
-0.1 & 2 & 0 & 0 \\
-2 & -0.1 & 0 & 0 \\
0 & 0 & -0.11 & 0 \\
0 & 0 & 0 & -2
\end{array}\right)
$$

Then, $\|(C+D)\|>1$,

and

$$
\lambda_{1,2}(C+D)=-0.1 \pm 2 j, \lambda_{3}(C+D)=-0.11, \lambda_{4}(C+D)=-2 .
$$

and

$$
0.5 \pi<\left|\arg \lambda_{i}(C+D)\right| \leq \pi(i=1,2,3,4)
$$

Now, two conditions are all satisfied, the $\delta$ synchronization between system (19) and system (20) can be realized.

The initial conditions were set as $\left(x_{1}, x_{2}, x_{3}, x_{4}\right)=(0,0,0.001,0.24)$, and $\|e(0)\|=1$.
Choose $k_{0}=10^{-3}$, and $\delta=k_{0}\|e(0)\|=1 \times 10^{-3}$. The curve of $k(t)$ with time $t$ is shown in Fig.2.

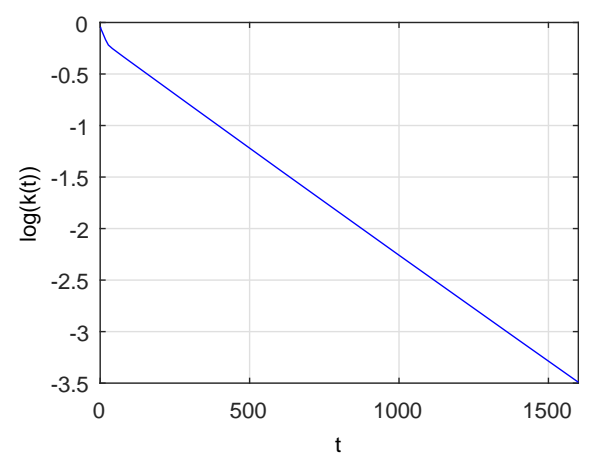

FIGURE II. $K(t)$ CHANGING WITH TIME $t$

In Fig.2, we can get when $t>1361 s, \log k(t) \leq k_{0}$. This result indicates that $\|e(t)\| \leq \delta=k_{0}\|e(0)\|=1 \times 10^{-3}$. Thus, the $\delta$ synchronization of system (19) and system (20) could be realized. The time response of error system is shown in Fig.3.

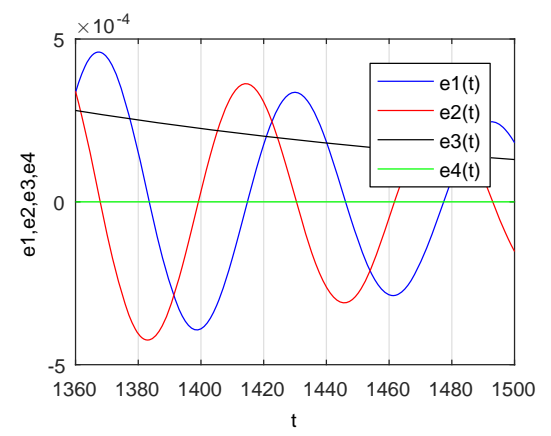

FIGURE III. THE TIME RESPONSE OF $\delta$ SYNCHRONIZATION ERROR FOR $\delta=k_{0}\|e(0)\|=1 \times 10^{-3}$

\section{CONCLUSION}

In this paper a $\delta$ synchronization approach is used for hyperchaotic system synchronization. Mathematical proof is given to show the theoretical feasibility of the method. A memristive hyperchaotic system is used to verify the effectiveness of this method. The simulations results indicate that this synchronization method is work well and suitable for actual system.

\section{ACKNOWLEDGMENT}

This work is supported by the National Natural Science Foundation of China (Grant No.51407012) and the Nature Science Foundation of Shaanxi Province under NO.2016JQ5047.

\section{REFERENCES}

[1] O. E., Rossler. An equation for hyperchaos. Physics Letters A, 1979,71, 155-157. 
[2] V. S., Udaltsov, J. P., Goedgebuer, L., Larger, J. B., Cuenot, P., Levy, and W. T., Rhodes. Communicating with hyperchaos:the dynamics of a dnlf emitter and recovery of transmitted information, 2003, Optics and Spectroscopy. 95,114

[3] S., Sadoudi, C., Tanougast, M. S., Azzaz, and A., Dandache. Design and FPGA implementation of a wireless hyperchaotic communication system for secure realtime image transmission. 2013, EURASIP Journal on Image and Video Processing, 43, 1.

[4] Y., Huang and X., Yang. Hyperchaos and bifurcation in a new class of four-dimensional Hopfield neural networks. 2006, Neurocomputing, 69, 1787.

[5] Samuel, G. Better memory, 2016, Commun. ACM, 59, 23.

[6] R., Vicente, J., Daud' en, P., Colet, and R., Toral. Analysis and characterization of the hyperchaos generated by a semiconductor laser subject to a delayed feedback loop. 2005, IEEE Journal of Quantum Electronics, 41, 541.

[7] Chua, L.O. Memristors-the missing circuit element. 1971, IEEE Trans. Circuit Theory, 18, 507.

[8] Zhou, L., Wang, C. H. and Zhou, L. L. Generating hyperchaotic multiwing attractor in a 4D memristive circuit. 2016, Nonlin. Dyn. 85, 2653.

[9] Ma, J., Chen, Z. Q., Wang, Z. L. and Zhang, Q.Four-wing hyperchaotic attractor generated from a $4 \mathrm{D}$ memristive system with a line equilibrium. 2015, Nonlin. Dyn. 81, 1275.

[10] L Zhou, C. H. Wang and L. L. Zhou. Generating Four-Wing Hyperchaotic Attractor and Two-Wing, Three-Wing, and Four-Wing Chaotic Attractors in 4D Memristive System. 2017, Int. J. Bifur. Chaos, $27,1750027$.

[11] H. Zhang and X. Ma. Synchronization of uncertain chaotic systems with parametersperturbation via active control. 2004, Chaos Solitons Fractals, $21,39$.

[12] J.S. Lin and J.J. Yan. Adaptive synchronization for two identical generalized Lorenzchaotic systems via a single controller. 2009, Nonlinear Anal. Real World Appl, 10, 1151.

[13] R.H. Li. Exponential generalized synchronization of uncertain coupled chaoticsystems by adaptive control. 2009, Commun. Nonlinear Sci. Numer. Simul. 14, 2757.

[14] G.P. Jiang, G.R. Chen, W.K. Tang. A new criterion for chaos synchronization usinglinear state feedback control. 2003, Int. J. Bifur. Chaos, 13, 2343.

[15] D. Lin, X. Wang, F. Nian, Y. Zhang. Dynamic fuzzy neural networks model-ing and adaptive backstepping tracking control of uncertain chaotic systems. 2010, Neurocomputing, 73, 2873. 